\title{
De-Rationalising Delusions
}

\author{
Authors: \\ Vaughan Bell, ${ }^{1,2}$ Nichola Raihani, ${ }^{3}$ Sam Wilkinson ${ }^{4}$
}

1. Research Department of Clinical, Educational and Health Psychology, University College London

2. Psychological Interventions Clinic for outpatients with Psychosis (PICuP), South London and Maudsley NHS Foundation Trust

3. Department of Experimental Psychology, University College London

4. Department of Sociology, Philosophy and Anthropology, Exeter University 


\begin{abstract}
Due to the traditional conceptualisation of delusion as 'irrational belief', cognitive models of delusions largely focus on impairments to domain-general reasoning. Nevertheless, current rationality-impairment models do not account for the fact that i) equivalently irrational beliefs can be induced through adaptive social cognitive processes, reflecting social integration rather than impairment; ii) delusions are overwhelmingly socially-themed; iii) delusions show a reduced sensitivity to social context, both in terms of how they are shaped and how they are communicated. Consequently, we argue that models of delusions need to include alteration to coalitional cognition - processes involved in affiliation, group perception, and the strategic management of relationships. This approach has the advantage of better accounting for both content (social themes) and form (fixity) of delusion. It is also supported by the established role of mesolimbic dopamine in both delusions and social organisation, and the ongoing reconceptualisation of belief as serving a social organisational function.
\end{abstract}


Delusions are defined as wildly improbable beliefs that are strongly held despite incontrovertible and obvious counter-evidence and despite what almost everyone else believes (American Psychiatric Association, 2013). Although the precise definition of delusions has been much debated (Bell, Halligan, \& Ellis, 2003; Coltheart, Langdon, \& McKay, 2011; David, 1999) they are considered the archetypal characteristic of 'madness' and a central component in the diagnosis of psychotic disorders such as schizophrenia, delusional disorder and schizoaffective disorder, as well as emerging after a range of neurological disorders and alterations to the central nervous system (Gilleen \& David, 2005).

Dominant historical and contemporary approaches have conceptualised delusions as pathological beliefs that are characterised by their irrationality (Bayne, 2017; Berrios, 1991; Bortolotti, 2005; Sakakibara, 2016). Consequently, current cognitive theories have largely focused on describing impairments to general-purpose inferential reasoning as the sole or central part of the explanatory model of delusions (Bell, Halligan, \& Ellis, 2006; Broyd, Balzan, Woodward, \& Allen, 2017; Feeney, Groman, Taylor, \& Corlett, 2017). Existing cognitive models have variously attempted to explain this through multi-factorial impairments to probabilistic reasoning, perception and affect (Garety \& Freeman, 1999), a two-stage impairment to perception and reasoning (Langdon \& Coltheart, 2000), aberrations in the use of prediction error in hierarchical inference mechanisms (Corlett, Taylor, Wang, Fletcher, \& Krystal, 2010), or impairments to various aspects of metacognitive representation and control (Bronstein, Pennycook, Joormann, Corlett, \& Cannon, 2019; Frith, Blakemore, \& Wolpert, 2000; Moritz \& Woodward, 2006)

However, we will argue here that this characterisation - that privileges the explanation of irrationality at the expense of explaining the social form of delusions - has hindered our understanding. Specifically, these approaches have focussed on finding problems with the components of domain-general rationality and have largely disregarded the potential role of socio-cognitive dysfunction. This approach has only seemed viable by failing to consider that healthy and adaptive social processes can form and maintain delusion-like beliefs, by ignoring the most striking phenomenological characteristics of delusions - namely that they are overwhelmingly socially and relationally themed, and by disregarding the fact that delusions show a reduced sensitivity to social context, both in terms of how they are shaped and how they are communicated. 
In line with recent work on the social organisational function of belief (Boyer, Firat, \& van Leeuwen, 2015; Echterhoff, Higgins, \& Levine, 2009; Gelpi, Cunningham, \& Buchsbaum, 2019; Jost, Ledgerwood, \& Hardin, 2008; Mercier \& Sperber, 2011), we suggest that causal models of delusions need to include dysfunction to coalitional cognition - that is, social cognitive processes involved in social influence, affiliation, interaction with groups, and the management of relationships. This approach has the advantage of better accounting for aspects of both the form and content of delusional beliefs as well as better reflecting the known function of the mesolimbic dopamine system - which is involved in the formation and maintenance of delusions and the management of social hierarchy, dominance and cooperation.

\section{The missing irrationality in delusions}

On face value, it might be reasonably assumed that someone who is distressed and disabled by the belief that they are dead (Sahoo \& Josephs, 2017), or that a camera has been implanted in their tooth and is taking pictures of their mind (Tanenberg-Karant et al., 1995), or that they are being poisoned by the CIA because they are Jesus (Mitchell \& Vierkant, 1991) has a problem with rationality.

Although some approaches have suggested delusions are solely explained by a problem with perception (Maher, 1999) or language (Hinzen, Rosselló, \& McKenna, 2016), the majority of cognitive accounts draw on the long-established historical idea that delusions reflect an impairment to rationality (Berrios, 1991) which now forms one of the central tenants of explanation for delusional beliefs (Bortolotti, 2010; Gold \& Hohwy, 2000; Leeser \& O’Donohue, 1999; Radden, 1985). This approach suggests that the presence of marked epistemic irrationality (i.e. beliefs that wildly deviate from the conclusions generated by normative principles of reasoning given available information), usually in addition to marked procedural rationality (i.e. inconsistency with clearly relevant and available pre-existing beliefs and mental states given the application of reliable forms of inference) (Bermúdez, 2001), implies problems with the cognitive processes underlying the capacity to reason pragmatically (Gerrans, 2001). Notably, the standard of comparison is normative because cognitive biases are considered to be an integral part of healthy, adaptive cognitive functioning (Bayne, 2017; Johnson, Blumstein, Fowler, \& Haselton, 2013). Consequently, 
this has motivated researchers to look for differences in performance on reasoning tasks that are assumed to reflect underlying impairments in domain-general rationality (Garety, 1991).

Initial assumptions that delusional beliefs reflect an impairment in syllogistic (i.e. deductive) reasoning (Von Domarus, 1944) have been discredited as numerous studies have failed to find any generalised difficulty in syllogistic reasoning associated with delusional beliefs when compared to IQ matched controls (Mujica-Parodi, Malaspina, \& Sackeim, 2000). Indeed, this is clearly apparent during conversation with someone with delusions. All things being equal, they are able to generate normative conclusions about matters not relating to their delusional beliefs, and only come to seemingly atypical conclusions when reasoning relates to the specific focus of the delusions. Therefore, most people with delusions appear not to have a generalised problem with rationality but a circumscribed irrationality, where conclusions are irrational solely in relation to the delusional content (Gold \& Hohwy, 2000).

It could be argued that one potential source of impairment to rationality might arise from general cognitive difficulties that are common in patients diagnosed with psychotic disorders (Bora, Yücel, \& Pantelis, 2010). However, there is only a weak correlation between general cognitive performance and the presence of positive symptoms in patients - of which delusions are a central feature (Ventura, Wood, \& Hellemann, 2013) and a significant effect of referral bias, in that community epidemiological studies show much smaller cognitive differences between people with and without psychosis than clinical studies (Mollon et al., 2016). Furthermore, cognitive impairment is neither necessary nor sufficient for the production of delusions as the vast majority of patients with even quite severe cognitive difficulties, for example after traumatic brain injury, do not develop delusions as a consequence, and indeed do not show rates that markedly differ from the general population (Ponsford, Alway, \& Gould, 2018). Clearly, some delusions arise after brain injury, dementia, alterations to the nervous system and so on, but there are no reliable instances of cognitive impairment independent of aetiology that reliably raise the risk of developing delusions.

One potential explanation for this is that delusions involve a more subtle type of cognitive alteration that is detectable but must interact with other difficulties, such as perceptual dysfunction, emotional dysregulation, traumatic life events and maladaptive coping to form 
and maintain delusional belief (e.g. (Garety, Kuipers, Fowler, Freeman, \& Bebbington, 2001; Langdon \& Coltheart, 2000).

Consequently, various cognitive biases have been proposed as important in the development or maintenance of delusions. The most studied has been the 'jumping to conclusions' probabilistic reasoning bias, most commonly tested using the 'beads task' (Garety, 1991; Huq, Garety, \& Hemsley, 1988). One recent large-scale study of people with first-episode psychosis reported that the 'jumping to conclusions' bias was reliably associated with psychosis (Tripoli et al., 2019) although this was entirely accounted for by general cognitive difficulties and not the presence of psychotic symptoms per se. Recent meta-analyses (Dudley, Taylor, Wickham, \& Hutton, 2016; So, Siu, Wong, Chan, \& Garety, 2016) reported that patients with current delusions draw fewer beads than patients without but this effect when quantified is 0.6 fewer beads; and $40-50 \%$ of patients showed no evidence of the bias whatsoever. Other cognitive biases associated with delusions show a similar pattern. A recent meta-analysis (McLean, Mattiske, \& Balzan, 2017) of a range of cognitive biases in delusions, including jumping to conclusions bias, bias against disconfirmatory evidence, bias against confirmatory evidence, and liberal acceptance bias, show small to moderate effect sizes of when comparing patients with and without schizophrenia, but did not include controls for general cognitive function.

We note here that research motivated by the search for evidence of problems in domaingeneral rationality has had some success, although the effects are relatively small in light of the striking deviations from normative conclusions about reality represented by the presence of delusions, suggesting an ongoing 'validity gap' in the rationality-impairment approach.

\section{Delusion-like beliefs arise from unimpaired, adaptive social processes}

In rationality-impairment approaches, the irrationality of beliefs is typically evaluated in terms of normative conclusions about the natural world (i.e. in terms of epistemic irrationality) and not in terms of the role the belief plays in the social environment. For example, it may be 'irrational' given the natural world for an individual to believe their thoughts control the weather but socially beneficial given that, if this belief is strongly held by their social group, rejecting it may lead to hostility and ostracism with potentially far 
greater negative consequences (Edis \& Boudry, 2019; Rauwolf, Mitchell, \& Bryson, 2015), suggesting it may be 'functionally rational' (Bayne, 2017).

Indeed, there are many examples of how strongly-held, affectively loaded, epistemically irrational beliefs can be reliably formed through social influence - demonstrating that adaptive social processes, rather than individual impairments in rationality, are sufficient to form delusion-like beliefs in cognitively intact people. We document several examples below.

The sudden spread of intense delusion-like ideas through the population are well-documented and frequently occur over time and across cultures and have variously been called 'mass delusion', 'social delusion' and have been studied alongside 'mass hysteria' (Bartholomew, 2001). Episodes of socially-transmitted delusion-like belief outbreaks have included beliefs in a 'phantom anaesthetist' who was believed to be prowling the community and 'gassing' members of the public (Bartholomew \& Victor, 2004), a 'windshield pitting epidemic' attributed to non-existent 'H-bomb tests' (Medalia \& Larsen, 1958), frequent waves of beliefs about malicious penis stealing episodes (Dan, Mondal, Chakraborty, Chaudhuri, \& Biswas, 2017), the kidnapping of children to use their decapitated heads in the foundations of new buildings (Barnes, 1993), and an invasion of foreign airships, before capable technology was available (Holman, 2016) to name but a few. Notably, these are distinct from conspiracy theories in that rather than being "explanations for important events that involve secret plots by powerful and malevolent groups" (Douglas, Sutton, \& Cichocka, 2017) they typically involve new and idiosyncratic phenomena that reflect a direct and personal risk to the individual believer. These episodes of 'mass social delusion' typically include thousands of people and motivate urgent behaviour designed to protect the individual and community but resolve quickly, usually within weeks, as the community interest wanes (Bartholomew, 2001)

Intense delusion-like beliefs that greatly deviate from both consensual reality and orthodoxy have been reported in members of small, isolated religious groups. These groups can be cults, which can involve coercive control (Rodríguez-Carballeira et al., 2015), but also more benign groups where membership is through enthusiastic and voluntary participation (Dawson, 1996). However, common to both is intense within-group contact and the reduction of extragroup social ties (Coates, 2012). Examples of firmly and intensely held, improbable, 
behaviour-shaping beliefs in members of non-coercive isolated religious groups include that mass suicide (eventually carried out) would allow group members to meet an alien space ship accompanying the Hale-Bopp comet which would cause them to ascend to "The Evolutionary Level Above Human" (Robinson, 1997), that a forthcoming nuclear war would wipe out a third of the world's population and the group members would be subsequently gifted miraculous powers of healing to save humanity (Melton, 1985), that God would appear on a specific cable TV channel to announce his presence to the world (Prather, 1999) and that group members were under attack from electromagnetic wave warfare designed to disrupt their spiritual work which could be deflected by wrapping themselves in white cloth (Murguia, 2011).

The transfer of delusional beliefs from one person to others has been variously classified as folie à deux, induced delusional disorder, and shared psychotic disorder (Shimizu, Kubota, Toichi, \& Baba, 2007). This can take several forms but the type of most relevance here is where a secondary person who is not otherwise experiencing psychosis takes on the delusional beliefs of the first person who has psychosis. It is now recognised that many secondary subjects of induced delusional disorder may risk factors for psychotic disorder genetic risk factors due to relatedness being key (Arnone, Patel, \& Tan, 2006). However, there are also clear cases of induced delusional disorder in secondaries who do not share relevant risk factors, including taking on the delusion of a primary with dementia that the house was being continually burgled by neighbours who would replace the items shortly after stealing them (Brooks, 1987), a husband, two sons, sister-in-law and nephew taking on a delusion that the neighbours were using technology to persecute them through the ceiling (Dippel, Kemper, \& Berger, 1991), and parents taking on the delusion resulting from amphetamine psychosis of the son that he was being persecuted, his walls were bugged, and he could detect which bricks had listening devices in them because of 'their different look' (Hill, Kevin, Patkar, \& Ashwin, 2001). Relevant non-biological risk factors for induced delusions include social isolation (José \& Mary, 1995), the length and closeness of the relationship (Arnone et al., 2006) and enmeshed and dependent relationship style (Mentjox, van Houten, \& Kooiman, 1993).

Experimentally, delusional beliefs have been modelled, albeit transiently, in the lab using hypnosis which involves a combination of suggestion and active engagement by highly 
suggestible individuals (Cox \& Barnier, 2010). More prosaically, although orthodox religious beliefs are complex and include dogma relating to social organisation as well as reality, they have a poor historical track record of reflecting accurate models of the natural world but have been successfully transmitted to large numbers of people (Edgell, 2012).

These examples demonstrate that healthy and adaptive social processes have a powerful role in belief formation - to the point where social influence can form and maintain beliefs that are as epistemically irrational, affectively loaded, and strongly-held as delusional beliefs. However, the fact that these beliefs wane or modify as the social context changes, and the fact that these beliefs reflect successful social integration in context of the community or micro-community in which they originate rather than social disability, suggests they reflect adaptive rather than impaired functioning. Therefore, these examples also demonstrate that privileging irrationality is unlikely to be an effective explanatory strategy for models of delusional belief, because it will not successfully account for their maladaptive nature. 


\section{The majority of delusions have social themes}

Delusions have a relatively select number of themes with social themes being overwhelmingly the most common presentation. Despite this clear social phenomenology, the majority of cognitive models of delusions do not consider it a focus for, nor a constraint on, explanation.

Common delusional themes include persecution, reference, guilt or sin, grandiosity, erotomania, jealousy, somatic changes, religion, mind reading, external control, thought broadcast, insertion and withdrawal (Gutiérrez-Lobos, Schmid-Siegel, Bankier, \& Walter, 2001; Paolini, Moretti, \& Compton, 2016; Peralta \& Cuesta, 2016). Delusions of persecution, reference, jealousy and erotomania are social as, by definition, they implicate social actors. Other delusion types are not by definition socially themed, but commonly present as social. Grandiose delusions often involve beliefs about having an elevated social position, important social role or link to important people (Suhail \& Cochrane, 2002). Delusions of guilt or sin typically involve concerns about contravening the expectations of others, including spiritual beings (Stompe et al., 1999). Religious delusions often involve a spiritual identity determining relationships with others, or a closeness or relationship with spiritual figures (Rieben et al., 2013). Delusions of external control, thought insertion, broadcast and withdrawal typically involves concerns about the social permeability of the private mental experience (Schimansky, Rössler, \& Haker, 2012) and being controlled by others (Hirjak, Breyer, Thomann, \& Fuchs, 2013). Delusions as a whole also commonly include beliefs about illusory social agents that seem present across themes (Bell, Mills, Modinos, \& Wilkinson, 2017).

Epidemiologically, persecutory delusions are by far the most common presentation in nonaffective psychosis (de Portugal et al., 2013; Ellersgaard et al., 2014), psychosis in Parkinson's disease (Warren, O'Gorman, Hume, Kisely, \& Siskind, 2018) and drug-induced psychoses (Voce, Calabria, Burns, Castle, \& McKetin, 2019). In the affective psychoses, grandiose delusions or delusions of guilt or shame may be more common depending on mood state (Picardi, Fonzi, Pallagrosi, Gigantesco, \& Biondi, 2018). Indeed, one of the notable attributes of delusions as a whole is that the majority involve key themes likely to reflect adaptive social processes shaped by evolution - for example, danger from others, social position, social stigma and social affiliation (Bortolotti, 2015; McKay \& Dennett, 2009). 
Despite this, the majority of cognitive models do not attempt to address why delusions are typically socially- rather than non-socially-themed. Several cognitive models exclude specific alterations to social cognition and do not address the social theming of delusions (Bronstein et al., 2019; Broyd et al., 2017; Feeney et al., 2017; Frith et al., 2000), several suggest that content, including social content, is determined by anomalous experience of which only specific examples are given (e.g. reduced autonomic responding to familiar people in Capgras delusion) which cannot account for the prevalence of social themes in delusions more generally (Langdon \& Coltheart, 2000; McKay, 2012) while others restrict themselves to a specific social theme, typically persecutory delusions (Bentall, Corcoran, Howard, Blackwood, \& Kinderman, 2001; Freeman, 2016), and so cannot explain why delusions per se have social rather than non-social themes

It is therefore clear that current cognitive models of delusional belief do not attempt to, or cannot, account for the most common thematic content of delusional beliefs.

\section{Delusions as dysfunction in coalitional cognition}

Based on i) the modest findings from studies searching for domain-general impairments to rationality; ii) the demonstrable formation of delusion-like irrational beliefs from healthy and adaptive social processes, and; iii) the social themes of the vast majority of delusions, we suggest that social cognitive processes are an important candidate for inclusion in any adequate cognitive model of delusions. This raises the question of how to conceptualise the role of social cognition in such models.

One potential objection to the inclusion of social cognitive mechanisms in models of delusions is that traditional measures of social cognition barely predict the presence of reality distortion symptoms of psychosis, tending to predict negative symptoms more strongly (Ventura et al., 2013). However, we agree with previous authors that the most widely used measures of social cognition do not capture the full range of relevant cognitive processes needed to explain disturbed social experience in psychopathology (Gallagher \& Varga, 2015; Schilbach, 2016; Yager \& Ehmann, 2006). Indeed, traditional measures tend to require individual testing of participants while exposing them to short instances of static social information that largely quantify individualised mechanisms of social information processing 
(Green, Horan, \& Lee, 2015) rather than the management of dynamic, evolving, multi-agent social situations (Bell et al., 2017; Schilbach, 2016).

Instead, we suggest that processes involved in social influence, affiliation and strategic social behaviour are likely to be key in understanding delusions. Cognitive processes focused on garnering support from other individuals, organising and maintaining alliances, and perceiving social status in groups have been described as 'coalitional psychology' something that is highly likely to be part of evolved cognitive system adapted to life in complex social groups (Boyer et al., 2015; Tooby \& Cosmides, 2010). Evidence for the evolutionary basis of this system stem from the fact that life in complex social groups favours the evolution of specialised and sophisticated socio-cognitive abilities (Dunbar R. I. M. \& Shultz Susanne, 2017) and that the ability to form and maintain coalitional alliances are present in multiple species and throughout hominid evolutionary history (Kappeler \& van Schaik, 2002).

We cite coalitional cognition as important in delusions for several reasons. Firstly, beliefs as a whole are now increasingly recognised as strongly or primarily social in function, implying that delusions need to be understood in terms of the same social mechanisms. Recent work in social cognition has suggested that conscious access to metacognitive judgements such as beliefs exists largely, if not primarily, to facilitate social interaction and group coordination (Tomasello, 2009). Frith (Frith, 2012b) describes how complex social coordination requires explicit communication of metacognitive judgements that allows for group refinement of knowledge and joint action. Similarly, Mercier and Sperber (Mercier \& Sperber, 2011) have argued that the primary function of reasoning is not for the refinement of personal knowledge, due to extensive evidence for how ineffective it is for this purpose, but for argumentation, social communication and persuasion. Here, beliefs, reasons, and knowledge are primarily generated to communicate what are usually intuitive inferences for group refinement and social coordination. Cushman argues (Cushman, 2019) that the post-hoc generation of reasons may not be efficient in itself for solving problems but it allows us to apply the same reflective process to ourselves and to others, again facilitating social coordination for problem-solving. 
Secondly, the social themes of delusions are also primarily coalitional in nature and involve concerns about persecution by others, social status, social affiliation or social transgressions (e.g. (McKay \& Dennett, 2009; Raihani \& Bell, 2019)). In addition, delusions frequently involve belief in the existence and actions of illusory social agents that impinge on the life of the believer (Bell et al., 2017). Indeed, we note that delusions are frequently delusional precisely because they cite the actions of coalitions who do not exist or who cannot perform the actions believed of them. Moreover, observational studies report that delusions typically involve coalitional characteristics that go often beyond the simple theme of the delusion itself. Individuals with persecutory delusions rank their perceived persecutors as having higher social rank than them (Green et al., 2006; Paget \& Ellett, 2014) and frequently involve a perception of being targeted by a specific group (Raihani \& Bell, 2019). Erotomanic delusions frequently involve the belief that a person of higher social status is in love with the believer (Brüne, 2001) while delusions of jealousy typically relate to perceived infidelity and the resultant humiliation (Seeman, 2016). Grandiose delusions involve a belief that the individual has risen in social status and has a special mission or message for the world (Knowles, McCarthy-Jones, \& Rowse, 2011). Experimental studies of psychosis and the psychosis-spectrum that have examined coalitional perception and behaviour have reported alterations in the social perception of groups as paranoia increases (Greenburgh, Bell, \& Raihani, 2019), an impact of in-group / out-group status on attribution of harmful intent in paranoia (Saalfeld, Ramadan, Bell, \& Raihani, 2018), alterations to the social representation of others (Raihani \& Bell, 2017), and a range of alterations to cooperative behaviour and emotional reactions to differences in cooperative behaviour in others accompanying delusions (Ellett, Allen-Crooks, Stevens, Wildschut, \& Chadwick, 2013; Fett et al., 2012, 2016; Gromann et al., 2013; Savulich et al., 2018).

Thirdly, by definition, delusions are both irrational and show reduced sensitivity to social context. This latter characteristic suggests they may involve a dysfunction in the ability to have beliefs moderated and refined through social interaction, and reflect problems with the strategic presentation of beliefs given social context. The criterion that delusions are not beliefs "ordinarily accepted by other members of the person's culture or subculture" has been maligned as a pragmatic decision to avoid the uncomfortable situation of diagnosing religious or spiritual beliefs as delusional due to the fact that many such beliefs would otherwise fulfil the irrationality criteria (Pierre, 2001; Ross \& McKay, 2017). But although religious beliefs 
may be epistemically irrational when judged against how well they explain the natural world they may still be highly socially beneficial. In contrast, delusions are typically epistemically irrational beliefs that, in addition, are socially maladaptive, indicated by the fact they form outside of a shared community of belief, are resistant to social context, leading to high levels of social disability and ostracism. Social context effects are not solely about the influence of the social milieu on the content and conviction in the belief, but also include being able to strategically communicate the belief given the social environment (Fransen, Smit, \& Verlegh, 2015). For example, one potential solution to having a belief that leads to social sanction is to keep it to oneself, to deny it, or to reveal it only to people who are unlikely to sanction you (Edis \& Boudry, 2019). Although not well studied, this is an ability people with delusions seem to progressively lose as delusions becomes more intense, as suggested by greater difficulties in using social context for pragmatic communication with increasing severity (Colle et al., 2013).

Finally, a coalitional approach to delusions is consistent with the known role of the mesolimbic dopamine system which has been strongly implicated in both delusions (Howes \& Kapur, 2009) and coalitional cognition (O’Connell \& Hofmann, 2011; Salamone \& Correa, 2012; Trainor, 2011). Increased dopamine turnover in the mesolimbic dopamine system is present in in patients with delusions (Winton-Brown, Fusar-Poli, Ungless, \& Howes, 2014) while increasing dopamine turnover by the use of stimulant drugs causes delusions after chronic use (Voce et al., 2019). These same drugs also increase social motivation and sensitivity to emotional expression in non-psychotic individuals (Wardle \& de Wit, 2012; Wardle, Garner, Munafò, \& de Wit, 2012). Conversely, antagonism of $\mathrm{D}_{2}$ dopamine receptors in the mesolimbic pathway by antipsychotic medication reduces the intensity of delusional beliefs (Kaar, Natesan, McCutcheon, \& Howes, 2019) and reduces social engagement and induces indifference (Gerlach \& Larsen, 1999; Moritz, Andreou, Klingberg, Thoering, \& Peters, 2013). Furthermore, a wealth of evidence from human and animal studies suggests that the mesolimbic dopamine system mediates various aspects of coalitional cognition, including perception and response to social hierarchy, social defeat and social dominance (Ghosal, Sandi, \& van der Kooij, 2019; Krach, Paulus, Bodden, \& Kircher, 2010; O'Connell \& Hofmann, 2011). Conversely, there is now increasing evidence that the risk of psychosis is raised by coalitional stress as social disadvantage leads to long-term 
changes in the mesolimbic dopamine pathway (Howes \& Kapur, 2009; Selten, van der Ven, Rutten, \& Cantor-Graae, 2013)

Thus, converging evidence from multiple sources, including observational and experimental studies of delusion, models of normal belief, the social responsiveness of delusional beliefs, and the functions of mesolimbic dopamine, suggest that coalitional cognition is likely an important, but currently overlooked, component of explanatory models of delusional belief.

\section{Conclusions and future directions}

We have argued that dysfunction to coalitional cognition is likely to be an important component in any adequate cognitive model of delusions. This approach has several benefits: i) it suggests how a model that includes dysfunction to social processes that moderate belief formation could better explain aspects of the form (fixity and reduced sensitivity to social context) and content (social themes) of delusions; ii) it distinguishes delusional from nondelusional epistemically irrational beliefs; iii) it is supported by the role of the mesolimbic dopamine system in both delusions and coalitional cognition.

However, we note several outstanding issues. The first is the relationship between dysfunction to coalitional cognition and dysfunction to other cognitive systems. We suggest that dysfunction to coalitional cognition may be sufficient, in itself, to account for some delusions. Given that well-functioning and adaptive social processes can form delusion-like beliefs, dysfunction to this system may lead to misconstrual of the social environment causing delusions to form without the need for impairment to other cognitive systems. The exact content of the belief ("Madonna is in love with me") may well be a post-hoc rationalisation of a less specific social inference that, for example, a high status person has a romantic connection to me, with the same dysfunction making it difficult to moderate or socially test the specific belief after it has formed.

Alternatively, there may be an interaction between an impairment in coalitional cognition and impairment to other cognitive systems. Perhaps most convincing in this regard are delusions of alien control that are frequently socially themed but evidence suggests may also depend on dysfunction to mechanisms of action monitoring and execution (Blakemore, Wolpert, \& Frith, 2002; Frith, 2012a). Similarly, perceptual dysfunction and hallucination may provide 
additional content for the delusional belief. However, it is worth noting here that hallucinated voices, the most common hallucinations that accompany delusions have clear social content in the majority of cases (Bell, 2013; Bell et al., 2017; Woods, Jones, Alderson-Day, Callard, $\&$ Fernyhough, 2015). Similarly, visual hallucinations, also common in psychosis, are most commonly social in content (Collerton et al., 2016; van Ommen, van Laar, Cornelissen, \& Bruggeman, 2019). We do not assume all perceptual disturbances related to delusions will have clear social content but we note the strong thematic similarity, suggesting altered coalitional cognition may also affect perceptual experience.

Furthermore, although most delusions have a clear social theme, a minority do not. Delusional parasitosis, the delusional belief you are infested with parasites, can include delusions about other people as agents of infection but most cases in the literature simply report a belief about infestation accompanied with perceptual disturbance in the form of formication, a sensation of crawling under the skin (Campbell, Elston, Hawthorne, \& Beckert, 2019). Avoidance of infection and contamination have been cited as basic mechanisms underlying social stigma and disgust and this has been cited as a factor in delusional parasitosis (Kupfer \& Fessler, 2018) but it is certainly the case that delusional parasitosis does not seem to be a prima facie example of a socially-themed delusion. Similarly, somatic and apocalyptic delusions are not by definition social, although the extent to which these are best understood as reflecting coalitional themes requires further investigation.

In terms of scope, it is clear that future research needs to orient towards the coalitional features of delusions and should deploy methods that better capture coalitional processes effectively. Notably, most cognitive models of delusions have either been 'generalist' aiming to explain the formation and maintenance of all delusions without particular reference to their theme, or have restricted themselves to specific themes (e.g. paranoid, external control) in a way that precludes the need to explain why delusions in general have a social theme.

Research that takes a comparative approach to delusional themes is still lacking and we note some important and common delusional themes (such as grandiose delusions) have received remarkably little research attention (Knowles et al., 2011).

Furthermore, additional focus needs to be paid to the coalitional phenomenology of 
delusions. For example, we have noted that explanations of paranoid delusions mostly characterise them as 'delusional fears about harm to self' despite the fact that they frequently include concerns about being persecuted by specific groups (Raihani \& Bell, 2019). Delusions, and indeed, hallucinated voices, seem to frequently involve illusory social agents but this social aspect of the experience has been, until recently, markedly under-researched (Bell et al., 2017). Similarly, many current paradigms in social cognition research are poorly able to capture coalitional processes such as evaluating relationships, alliances, and tracking agent identity and status. Social cognition research in psychosis and schizophrenia has broadly coalesced on studying processes related to perception of individual others largely stemming from methods that test individuals by exposing them to social information or involving them in simulated social situations (Green et al., 2015). Paradigms that involve experimental studies of interaction between individuals (e.g. 'second person neuroscience' (Schilbach, 2016; Schilbach et al., 2013) or that examine interaction with groups (e.g. (Greenburgh et al., 2019) are likely to be key in capturing the mechanisms of social influence and alliance.

In summary, we argue for a move away from attempts to explain delusions based primarily in terms of individual irrationality and instead towards models that include dysfunction to coalitional cognition as an essential component, which we argue are urgently needed to better capture the known form, content, and mechanisms of delusional beliefs. 


\section{References}

American Psychiatric Association, A. P. (2013). Diagnostic and statistical manual of mental disorders (DSM-5®). American Psychiatric Pub.

Arnone, D., Patel, A., \& Tan, G. M.-Y. (2006). The nosological significance of Folie à Deux: A review of the literature. Annals of General Psychiatry, 5, 11. https://doi.org/10.1186/1744-859X-5-11

Barnes, R. H. (1993). Construction Sacrifice, Kidnapping and Head-hunting Rumors on Flores and Elsewhere in Indonesia. Oceania, 64(2), 146-158. https://doi.org/10.1002/j.1834-4461.1993.tb02459.x

Bartholomew, R. E. (2001). Little green men, meowing nuns and head-hunting panics: A study of mass psychogenic illness and social delusion. McFarland.

Bartholomew, R. E., \& Victor, J. S. (2004). A Social-Psychological Theory of Collective Anxiety Attacks: The "Mad Gasser” Reexamined. The Sociological Quarterly, 45(2), 229-248. Retrieved from JSTOR.

Bayne, T. (2017). Chapter 5-Delusion and the Norms of Rationality. In T.-W. Hung \& T. J. Lane (Eds.), Rationality (pp. 77-94). https://doi.org/10.1016/B978-0-12-804600$5.00005-2$

Bell, V. (2013). A Community of One: Social Cognition and Auditory Verbal Hallucinations. PLoS Biol, 11(12), e1001723. https://doi.org/10.1371/journal.pbio.1001723

Bell, V., Halligan, P. W., \& Ellis, H. (2003). Beliefs about delusions. The Psychologist, 16(8), 418-423.

Bell, V., Halligan, P. W., \& Ellis, H. D. (2006). Explaining delusions: A cognitive perspective. Trends in Cognitive Sciences, 10(5), 219-226. https://doi.org/10.1016/j.tics.2006.03.004 
Bell, V., Mills, K. L., Modinos, G., \& Wilkinson, S. (2017). Rethinking Social Cognition in Light of Psychosis: Reciprocal Implications for Cognition and Psychopathology. Clinical Psychological Science, 5(3), 537-550. https://doi.org/10.1177/2167702616677079

Bentall, R. P., Corcoran, R., Howard, R., Blackwood, N., \& Kinderman, P. (2001). PERSECUTORY DELUSIONS: A REVIEW AND THEORETICAL INTEGRATION. Clinical Psychology Review, 21(8), 1143-1192. https://doi.org/10.1016/S0272-7358(01)00106-4

Bermúdez, J. L. (2001). Normativity and Rationality in Delusional Psychiatric Disorders. Mind \& Language, 16(5), 493-457. https://doi.org/10.1111/1468-0017.00179

Berrios, G. E. (1991). Delusions as "Wrong Beliefs": A Conceptual History. The British Journal of Psychiatry, 159(S14), 6-13. https://doi.org/10.1192/S0007125000296414

Blakemore, S.-J., Wolpert, D. M., \& Frith, C. D. (2002). Abnormalities in the awareness of action. Trends in Cognitive Sciences, 6(6), 237-242. https://doi.org/10.1016/S13646613(02)01907-1

Bora, E., Yücel, M., \& Pantelis, C. (2010). Cognitive Impairment in Schizophrenia and Affective Psychoses: Implications for DSM-V Criteria and Beyond. Schizophrenia Bulletin, 36(1), 36-42. https://doi.org/10.1093/schbul/sbp094

Bortolotti, L. (2005). Delusions and the Background of Rationality. Mind \& Language, 20(2), 189-208. https://doi.org/10.1111/j.0268-1064.2005.00282.x

Bortolotti, L. (2010). Delusions and other irrational beliefs. Oxford University Press. Bortolotti, L. (2015). The epistemic innocence of motivated delusions. Consciousness and Cognition, 33, 490-499. https://doi.org/10.1016/j.concog.2014.10.005 
Boyer, P., Firat, R., \& van Leeuwen, F. (2015). Safety, Threat, and Stress in Intergroup Relations: A Coalitional Index Model. Perspectives on Psychological Science, 10(4), 434-450. https://doi.org/10.1177/1745691615583133

Bronstein, M. V., Pennycook, G., Joormann, J., Corlett, P. R., \& Cannon, T. D. (2019). Dualprocess theory, conflict processing, and delusional belief. Clinical Psychology Review, 72, 101748. https://doi.org/10.1016/j.cpr.2019.101748

Brooks, S. A. (1987). Folie à Deux in the Aged: Variations in Psychopathology. The Canadian Journal of Psychiatry, 32(1), 61-63. https://doi.org/10.1177/070674378703200112

Broyd, A., Balzan, R. P., Woodward, T. S., \& Allen, P. (2017). Dopamine, cognitive biases and assessment of certainty: A neurocognitive model of delusions. Clinical Psychology Review, 54(Supplement C), 96-106. https://doi.org/10.1016/j.cpr.2017.04.006

Brüne, M. (2001). De Clérambault's syndrome (erotomania) in an evolutionary perspective. Evolution and Human Behavior, 22(6), 409-415. https://doi.org/10.1016/S10905138(01)00077-0

Campbell, E. H., Elston, D. M., Hawthorne, J. D., \& Beckert, D. R. (2019). Diagnosis and management of delusional parasitosis. Journal of the American Academy of Dermatology, 80(5), 1428-1434. https://doi.org/10.1016/j.jaad.2018.12.012

Coates, D. D. (2012). “Cult Commitment” from the Perspective of Former Members: Direct Rewards of Membership versus Dependency Inducing Practices. Deviant Behavior, 33(3), 168-184. https://doi.org/10.1080/01639625.2010.548302

Colle, L., Angeleri, R., Vallana, M., Sacco, K., Bara, B. G., \& Bosco, F. M. (2013). Understanding the communicative impairments in schizophrenia: A preliminary 
study. Journal of Communication Disorders, 46(3), 294-308.

https://doi.org/10.1016/j.jcomdis.2013.01.003

Collerton, D., Tayor, J.-P., Tsuda, I., Fujii, H., Nara, S., Aihara, K., \& Katori, Y. (2016).

How Can We See Things That Are Not There?: Current Insights into Complex Visual Hallucinations. Journal of Consciousness Studies, 23(7-8), 195-227.

Coltheart, M., Langdon, R., \& McKay, R. (2011). Delusional Belief. Annual Review of Psychology, 62(1), 271-298. https://doi.org/10.1146/annurev.psych.121208.131622

Corlett, P. R., Taylor, J. R., Wang, X.-J., Fletcher, P. C., \& Krystal, J. H. (2010). Toward a neurobiology of delusions. Progress in Neurobiology, 92(3), 345-369. https://doi.org/10.1016/j.pneurobio.2010.06.007

Cox, R. E., \& Barnier, A. J. (2010). Hypnotic illusions and clinical delusions: Hypnosis as a research method. Cognitive Neuropsychiatry, 15(1-3), 202-232. https://doi.org/10.1080/13546800903319884

Cushman, F. (2019). Rationalization is rational. Behavioral and Brain Sciences, 1-69. https://doi.org/10.1017/S0140525X19001730

Dan, A., Mondal, T., Chakraborty, K., Chaudhuri, A., \& Biswas, A. (2017). Clinical course and treatment outcome of Koro: A follow up study from a Koro epidemic reported from West Bengal, India. Asian Journal of Psychiatry, 26, 14-20. https://doi.org/10.1016/j.ajp.2016.12.016

David, A. S. (1999). On the Impossibility of Defining Delusions. Philosophy, Psychiatry, \& Psychology, 6(1), 17-20.

Dawson, L. L. (1996). Who joins new religious movements and why: Twenty years of research and what have we learned? Studies in Religion/Sciences Religieuses, 25(2), 141-161. https://doi.org/10.1177/000842989602500202 
de Portugal, E., González, N., del Amo, V., Haro, J. M., Díaz-Caneja, C. M., de Dios Luna del Castillo, J., \& Cervilla, J. A. (2013). Empirical redefinition of delusional disorder and its phenomenology: The DELIREMP study. Comprehensive Psychiatry, 54(3), 243-255. https://doi.org/10.1016/j.comppsych.2012.08.002

Dippel, B., Kemper, J., \& Berger, M. (1991). Folie à six: A case report on induced psychotic disorder. Acta Psychiatrica Scandinavica, 83(2), 137-141. https://doi.org/10.1111/j.1600-0447.1991.tb07379.x

Douglas, K. M., Sutton, R. M., \& Cichocka, A. (2017). The Psychology of Conspiracy Theories. Current Directions in Psychological Science, 26(6), 538-542. https://doi.org/10.1177/0963721417718261

Dudley, R., Taylor, P., Wickham, S., \& Hutton, P. (2016). Psychosis, Delusions and the “Jumping to Conclusions" Reasoning Bias: A Systematic Review and Meta-analysis. Schizophrenia Bulletin, 42(3), 652-665. https://doi.org/10.1093/schbul/sbv150

Dunbar R. I. M., \& Shultz Susanne. (2017). Why are there so many explanations for primate brain evolution? Philosophical Transactions of the Royal Society B: Biological Sciences, 372(1727), 20160244. https://doi.org/10.1098/rstb.2016.0244

Echterhoff, G., Higgins, E. T., \& Levine, J. M. (2009). Shared Reality: Experiencing Commonality with others' Inner States about the World. Perspectives on Psychological Science, 4(5), 496-521. https://doi.org/10.1111/j.17456924.2009.01161.x

Edgell, P. (2012). A Cultural Sociology of Religion: New Directions. Annual Review of Sociology, 38(1), 247-265. https://doi.org/10.1146/annurev-soc-071811-145424

Edis, T., \& Boudry, M. (2019). Truth and Consequences: When Is It Rational to Accept Falsehoods? Journal of Cognition and Culture, 19(1-2), 147-169. https://doi.org/10.1163/15685373-12340052 
Ellersgaard, D., Mors, O., Thorup, A., Jørgensen, P., Jeppesen, P., \& Nordentoft, M. (2014).

Prospective study of the course of delusional themes in first-episode non-affective psychosis. Early Intervention in Psychiatry, 8(4), 340-347.

https://doi.org/10.1111/eip.12059

Ellett, L., Allen-Crooks, R., Stevens, A., Wildschut, T., \& Chadwick, P. (2013). A paradigm for the study of paranoia in the general population: The Prisoner's Dilemma Game. Cognition and Emotion, 27(1), 53-62. https://doi.org/10.1080/02699931.2012.689757

Feeney, E. J., Groman, S. M., Taylor, J. R., \& Corlett, P. R. (2017). Explaining Delusions: Reducing Uncertainty Through Basic and Computational Neuroscience. Schizophrenia Bulletin, 43(2), 263-272. https://doi.org/10.1093/schbul/sbw194

Fett, A.-K. J., Shergill, S. S., Joyce, D. W., Riedl, A., Strobel, M., Gromann, P. M., \& Krabbendam, L. (2012). To trust or not to trust: The dynamics of social interaction in psychosis. Brain, 135(3), 976-984. https://doi.org/10.1093/brain/awr359

Fett, A.-K. J., Shergill, S. S., Korver-Nieberg, N., Yakub, F., Gromann, P. M., \& Krabbendam, L. (2016). Learning to trust: Trust and attachment in early psychosis. Psychological Medicine, 46(7), 1437-1447. https://doi.org/10.1017/S0033291716000015

Fransen, M. L., Smit, E. G., \& Verlegh, P. W. J. (2015). Strategies and motives for resistance to persuasion: An integrative framework. Frontiers in Psychology, 6. https://doi.org/10.3389/fpsyg.2015.01201

Freeman, D. (2016). Persecutory delusions: A cognitive perspective on understanding and treatment. The Lancet Psychiatry, 3(7), 685-692. https://doi.org/10.1016/S22150366(16)00066-3 
Frith, C., Blakemore, S.-J., \& Wolpert, D. M. (2000). Explaining the symptoms of schizophrenia: Abnormalities in the awareness of action. Brain Research Reviews, 31(2), 357-363. https://doi.org/10.1016/S0165-0173(99)00052-1

Frith, C. D. (2012a). Explaining delusions of control: The comparator model 20years on. Consciousness and Cognition, 21(1), 52-54. https://doi.org/10.1016/j.concog.2011.06.010

Frith, C. D. (2012b). The role of metacognition in human social interactions. Philosophical Transactions of the Royal Society B: Biological Sciences, 367(1599), 2213-2223. https://doi.org/10.1098/rstb.2012.0123

Gallagher, S., \& Varga, S. (2015). Social cognition and psychopathology: A critical overview. World Psychiatry, 14(1), 5-14. https://doi.org/10.1002/wps.20173

Garety, P. (1991). Reasoning and Delusions. The British Journal of Psychiatry, 159(S14), 14-18. https://doi.org/10.1192/S0007125000296426

Garety, P. A., \& Freeman, D. (1999). Cognitive approaches to delusions: A critical review of theories and evidence. British Journal of Clinical Psychology, 38(2), 113-154. https://doi.org/10.1348/014466599162700

Garety, P. A., Kuipers, E., Fowler, D., Freeman, D., \& Bebbington, P. E. (2001). A cognitive model of the positive symptoms of psychosis. Psychological Medicine, 31(2), 189195. https://doi.org/10.1017/S0033291701003312

Gelpi, R., Cunningham, W. A., \& Buchsbaum, D. (2019). Belief as a Non-Epistemic Adaptive Benefit [Preprint]. https://doi.org/10.31234/osf.io/6ythf

Gerlach, J., \& Larsen, E. B. (1999). Subjective experience and mental side-effects of antipsychotic treatment. Acta Psychiatrica Scandinavica, 99(s395), 113-117. https://doi.org/10.1111/j.1600-0447.1999.tb05990.x 
Gerrans, P. (2001). Delusions as performance failures. Cognitive Neuropsychiatry, 6(3), 161173. https://doi.org/10.1080/1354680004200016

Ghosal, S., Sandi, C., \& van der Kooij, M. A. (2019). Neuropharmacology of the mesolimbic system and associated circuits on social hierarchies. Neuropharmacology. https://doi.org/10.1016/j.neuropharm.2019.01.013

Gilleen, J., \& David, A. S. (2005). The cognitive neuropsychiatry of delusions: From psychopathology to neuropsychology and back again. Psychological Medicine, 35(1), 5-12. https://doi.org/10.1017/S0033291704003976

Gold, I., \& Hohwy, J. (2000). Rationality and Schizophrenic Delusion. Mind \& Language, 15(1), 146-167. https://doi.org/10.1111/1468-0017.00127

Green, C., Garety, P. A., Freeman, D., Fowler, D., Bebbington, P., Dunn, G., \& Kuipers, E. (2006). Content and affect in persecutory delusions. British Journal of Clinical Psychology, 45(4), 561-577. https://doi.org/10.1348/014466506X98768

Green, M. F., Horan, W. P., \& Lee, J. (2015). Social cognition in schizophrenia. Nature Reviews Neuroscience, 16(10), 620-631. https://doi.org/10.1038/nrn4005

Greenburgh, A., Bell, V., \& Raihani, N. (2019). Paranoia and conspiracy: Group cohesion increases harmful intent attribution in the Trust Game. PeerJ, 7, e7403. https://doi.org/10.7717/peerj.7403

Gromann, P. M., Heslenfeld, D. J., Fett, A.-K., Joyce, D. W., Shergill, S. S., \& Krabbendam, L. (2013). Trust versus paranoia: Abnormal response to social reward in psychotic illness. Brain, awt076. https://doi.org/10.1093/brain/awt076

Gutiérrez-Lobos, K., Schmid-Siegel, B., Bankier, B., \& Walter, H. (2001). Delusions in FirstAdmitted Patients: Gender, Themes and Diagnoses. Psychopathology, 34(1), 1-7. https://doi.org/10.1159/000049273 
Hill, B., Kevin, P., Patkar, M., \& Ashwin, A. (2001). Folie a famille associated with amphetamine use. Jefferson Journal of Psychiatry, 16(1), 5.

Hinzen, W., Rosselló, J., \& McKenna, P. (2016). Can delusions be understood linguistically? Cognitive Neuropsychiatry, 21(4), 281-299. https://doi.org/10.1080/13546805.2016.1190703

Hirjak, D., Breyer, T., Thomann, P. A., \& Fuchs, T. (2013). Disturbance of Intentionality: A Phenomenological Study of Body-Affecting First-Rank Symptoms in Schizophrenia. PLOS ONE, 8(9), e73662. https://doi.org/10.1371/journal.pone.0073662

Holman, B. (2016). The Phantom Airship Panic of 1913: Imagining Aerial Warfare in Britain before the Great War. Journal of British Studies, 55(1), 99-119. https://doi.org/10.1017/jbr.2015.173

Howes, O. D., \& Kapur, S. (2009). The Dopamine Hypothesis of Schizophrenia: Version III-The Final Common Pathway. Schizophrenia Bulletin, 35(3), 549-562. https://doi.org/10.1093/schbul/sbp006

Huq, S. F., Garety, P. A., \& Hemsley, D. R. (1988). Probabilistic Judgements in Deluded and Non-Deluded Subjects. The Quarterly Journal of Experimental Psychology Section A, 40(4), 801-812. https://doi.org/10.1080/14640748808402300

Johnson, D. D. P., Blumstein, D. T., Fowler, J. H., \& Haselton, M. G. (2013). The evolution of error: Error management, cognitive constraints, and adaptive decision-making biases. Trends in Ecology \& Evolution, 28(8), 474-481. https://doi.org/10.1016/j.tree.2013.05.014

José, M. S., \& Mary, V. S. (1995). Shared Psychotic Disorder: A Critical Review of the Literature. The Canadian Journal of Psychiatry, 40(7), 389-395. https://doi.org/10.1177/070674379504000705 
Jost, J. T., Ledgerwood, A., \& Hardin, C. D. (2008). Shared Reality, System Justification, and the Relational Basis of Ideological Beliefs. Social and Personality Psychology Compass, 2(1), 171-186. https://doi.org/10.1111/j.1751-9004.2007.00056.x

Kaar, S. J., Natesan, S., McCutcheon, R., \& Howes, O. D. (2019). Antipsychotics: Mechanisms underlying clinical response and side-effects and novel treatment approaches based on pathophysiology. Neuropharmacology, 107704. https://doi.org/10.1016/j.neuropharm.2019.107704

Kappeler, P. M., \& van Schaik, C. P. (2002). Evolution of Primate Social Systems. International Journal of Primatology, 23(4), 707-740. https://doi.org/10.1023/A:1015520830318

Knowles, R., McCarthy-Jones, S., \& Rowse, G. (2011). Grandiose delusions: A review and theoretical integration of cognitive and affective perspectives. Clinical Psychology Review, 31(4), 684-696. https://doi.org/10.1016/j.cpr.2011.02.009

Krach, S., Paulus, F. M., Bodden, M., \& Kircher, Ti. (2010). The rewarding nature of social interactions. Frontiers in Behavioral Neuroscience, 4. https://doi.org/10.3389/fnbeh.2010.00022

Kupfer, T. R., \& Fessler, D. M. T. (2018). Ectoparasite defence in humans: Relationships to pathogen avoidance and clinical implications. Philosophical Transactions of the Royal Society of London. Series B, Biological Sciences, 373(1751). https://doi.org/10.1098/rstb.2017.0207

Langdon, R., \& Coltheart, M. (2000). The Cognitive Neuropsychology of Delusions. Mind \& Language, 15(1), 184-218. https://doi.org/10.1111/1468-0017.00129

Leeser, J., \& O’Donohue, W. (1999). What is a delusion? Epistemological dimensions. Journal of Abnormal Psychology, 108(4), 687-694. https://doi.org/10.1037/0021843X.108.4.687 
Maher, B. A. (1999). Anomalous Experience in Everyday Life: Its Significance for Psychopathology. The Monist, 82(4), 547-570. Retrieved from JSTOR.

McKay, R. (2012). Delusional Inference. Mind \& Language, 27(3), 330-355. https://doi.org/10.1111/j.1468-0017.2012.01447.x

McKay, R. T., \& Dennett, D. C. (2009). The evolution of misbelief. Behavioral and Brain Sciences, 32(06), 493-510. https://doi.org/10.1017/S0140525X09990975

McLean, B. F., Mattiske, J. K., \& Balzan, R. P. (2017). Association of the Jumping to Conclusions and Evidence Integration Biases With Delusions in Psychosis: A Detailed Meta-analysis. Schizophrenia Bulletin, 43(2), 344-354. https://doi.org/10.1093/schbul/sbw056

Medalia, N. Z., \& Larsen, O. N. (1958). Diffusion and Belief in a Collective Delusion: The Seattle Windshield Pitting Epidemic. American Sociological Review, 23(2), 180-186. https://doi.org/10.2307/2089002

Melton, J. G. (1985). Spiritualization and reaffirmation: What really happens when prophecy fails. American Studies, 26(2), 17-29.

Mentjox, R., van Houten, C. A. G., \& Kooiman, C. G. (1993). Induced psychotic disorder: Clinical aspects, theoretical considerations, and some guidelines for treatment. Comprehensive Psychiatry, 34(2), 120-126. https://doi.org/10.1016/0010440X(93)90056-A

Mercier, H., \& Sperber, D. (2011). Why do humans reason? Arguments for an argumentative theory. Behavioral and Brain Sciences, 34(2), 57-74. https://doi.org/10.1017/S0140525X10000968

Mitchell, J., \& Vierkant, A. D. (1991). Delusions and Hallucinations of Cocaine Abusers and Paranoid Schizophrenics: A Comparative Study. The Journal of Psychology, 125(3), 301-310. https://doi.org/10.1080/00223980.1991.10543294 
Mollon, J., David, A. S., Morgan, C., Frissa, S., Glahn, D., Pilecka, I., ... Reichenberg, A. (2016). Psychotic Experiences and Neuropsychological Functioning in a Populationbased Sample. JAMA Psychiatry, 73(2), 129-138. https://doi.org/10.1001/jamapsychiatry.2015.2551

Moritz, S., Andreou, C., Klingberg, S., Thoering, T., \& Peters, M. J. V. (2013). Assessment of subjective cognitive and emotional effects of antipsychotic drugs. Effect by defect? Neuropharmacology, 72, 179-186. https://doi.org/10.1016/j.neuropharm.2013.04.039

Moritz, S., \& Woodward, T. S. (2006). Metacognitive control over false memories: A key determinant of delusional thinking. Current Psychiatry Reports, 8(3), 184-190. https://doi.org/10.1007/s11920-006-0022-2

Mujica-Parodi, L. R., Malaspina, D., \& Sackeim, H. A. (2000). Logical Processing, Affect, and Delusional Thought in Schizophrenia. Harvard Review of Psychiatry, 8(2), 7383. https://doi.org/10.1080/hrp_8.2.73

Murguia, S. J. (2011). When Prophets Fail to Fail: A Case Study of Yuko Chino, Chino Shoho, and the Pana-Wave Laboratory. How Prophecy Lives, 21, 99.

O’Connell, L. A., \& Hofmann, H. A. (2011). The Vertebrate mesolimbic reward system and social behavior network: A comparative synthesis. Journal of Comparative Neurology, 519(18), 3599-3639. https://doi.org/10.1002/cne.22735

Paget, A., \& Ellett, L. (2014). Relationships Among Self, Others, and Persecutors in Individuals With Persecutory Delusions: A Repertory Grid Analysis. Behavior Therapy, 45(2), 273-282. https://doi.org/10.1016/j.beth.2013.12.001

Paolini, E., Moretti, P., \& Compton, M. T. (2016). Delusions in first-episode psychosis: Principal component analysis of twelve types of delusions and demographic and clinical correlates of resulting domains. Psychiatry Research, 243, 5-13. https://doi.org/10.1016/j.psychres.2016.06.002 
Peralta, V., \& Cuesta, M. J. (2016). Delusional disorder and schizophrenia: A comparative study across multiple domains. Psychological Medicine, 46(13), 2829-2839. https://doi.org/10.1017/S0033291716001501

Picardi, A., Fonzi, L., Pallagrosi, M., Gigantesco, A., \& Biondi, M. (2018). Delusional Themes Across Affective and Non-Affective Psychoses. Frontiers in Psychiatry, 9. https://doi.org/10.3389/fpsyt.2018.00132

Pierre, J. (2001). Faith or Delusion? At the Crossroads of Religion and Psychosis. Journal of Psychiatric Practice, 7(3), 163-172.

Ponsford, J., Alway, Y., \& Gould, K. R. (2018). Epidemiology and Natural History of Psychiatric Disorders After TBI. The Journal of Neuropsychiatry and Clinical Neurosciences, 30(4), 262-270. https://doi.org/10.1176/appi.neuropsych.18040093

Prather, C. H. (1999). God's Salvation Church: Past, Present and Future. Marburg Journal of Religion, 4(1). https://doi.org/10.17192/mjr.1999.4.3761

Radden, J. (1985). Madness and reason. Unwin Hyman.

Raihani, N. J., \& Bell, V. (2017). Paranoia and the social representation of others: A largescale game theory approach. Scientific Reports, 7(1), 1-9. https://doi.org/10.1038/s41598-017-04805-3

Raihani, N. J., \& Bell, V. (2019). An evolutionary perspective on paranoia. Nature Human Behaviour, 3(2), 114. https://doi.org/10.1038/s41562-018-0495-0

Rauwolf, P., Mitchell, D., \& Bryson, J. J. (2015). Value homophily benefits cooperation but motivates employing incorrect social information. Journal of Theoretical Biology, 367, 246-261. https://doi.org/10.1016/j.jtbi.2014.11.023

Rieben, I., Mohr, S., Borras, L., Gillieron, C., Brandt, P.-Y., Perroud, N., \& Huguelet, P. (2013). A Thematic Analysis of Delusion With Religious Contents in Schizophrenia: 
Open, Closed, and Mixed Dynamics. The Journal of Nervous and Mental Disease, 201(8), 665-673. https://doi.org/10.1097/NMD.0b013e31829c5073

Robinson, W. G. (1997). Heaven's Gate: The End. Journal of Computer-Mediated Communication, 3(3). https://doi.org/10.1111/j.1083-6101.1997.tb00077.x

Rodríguez-Carballeira, Á., Saldaña, O., Almendros, C., Martín-Peña, J., Escartín, J., \& Porrúa-García, C. (2015). Group psychological abuse: Taxonomy and severity of its components. The European Journal of Psychology Applied to Legal Context, 7(1), 31-39. https://doi.org/10.1016/j.ejpal.2014.11.001

Ross, R. M., \& McKay, R. (2017). Why is belief in God not a delusion? Religion, Brain \& Behavior, 7(4), 316-319. https://doi.org/10.1080/2153599X.2016.1249917

Saalfeld, V., Ramadan, Z., Bell, V., \& Raihani, N. J. (2018). Experimentally induced social threat increases paranoid thinking. Royal Society Open Science, 5(8), 180569. https://doi.org/10.1098/rsos.180569

Sahoo, A., \& Josephs, K. A. (2017). A Neuropsychiatric Analysis of the Cotard Delusion. The Journal of Neuropsychiatry and Clinical Neurosciences, appi.neuropsych.17010018. https://doi.org/10.1176/appi.neuropsych.17010018

Sakakibara, E. (2016). Irrationality and Pathology of Beliefs. Neuroethics, 9(2), 147-157. https://doi.org/10.1007/s12152-016-9256-9

Salamone, J. D., \& Correa, M. (2012). The Mysterious Motivational Functions of Mesolimbic Dopamine. Neuron, 76(3), 470-485. https://doi.org/10.1016/j.neuron.2012.10.021

Savulich, G., Jeanes, H., Rossides, N., Kaur, S., Zacharia, A., Robbins, T. W., \& Sahakian, B. J. (2018). Moral Emotions and Social Economic Games in Paranoia. Frontiers in Psychiatry, 9. https://doi.org/10.3389/fpsyt.2018.00615 
Schilbach, L. (2016). Towards a second-person neuropsychiatry. Phil. Trans. R. Soc. B, 371(1686), 20150081. https://doi.org/10.1098/rstb.2015.0081

Schilbach, L., Timmermans, B., Reddy, V., Costall, A., Bente, G., Schlicht, T., \& Vogeley, K. (2013). Toward a second-person neuroscience. Behavioral and Brain Sciences, 36(04), 393-414. https://doi.org/10.1017/S0140525X12000660

Schimansky, J., Rössler, W., \& Haker, H. (2012). The Influence of Social Cognition on Ego Disturbances in Patients with Schizophrenia. Psychopathology, 45(2), 117-125. https://doi.org/10.1159/000330264

Seeman, M. V. (2016). Pathological Jealousy: An Interactive Condition. Psychiatry, 79(4), 379-388. https://doi.org/10.1080/00332747.2016.1175838

Selten, J.-P., van der Ven, E., Rutten, B. P. F., \& Cantor-Graae, E. (2013). The Social Defeat Hypothesis of Schizophrenia: An Update. Schizophrenia Bulletin, 39(6), 1180-1186. https://doi.org/10.1093/schbul/sbt134

Shimizu, M., Kubota, Y., Toichi, M., \& Baba, H. (2007). Folie à Deux and shared psychotic disorder. Current Psychiatry Reports, 9(3), 200-205. https://doi.org/10.1007/s11920007-0019-5

So, S. H., Siu, N. Y., Wong, H., Chan, W., \& Garety, P. A. (2016). 'Jumping to conclusions' data-gathering bias in psychosis and other psychiatric disorders-Two meta-analyses of comparisons between patients and healthy individuals. Clinical Psychology Review, 46, 151-167. https://doi.org/10.1016/j.cpr.2016.05.001

Stompe, T., Friedman, A., Ortwein, G., Strobl, R., Chaudhry, H. R., Najam, N., \& Chaudhry, M. R. (1999). Comparison of Delusions among Schizophrenics in Austria and in Pakistan. Psychopathology, 32(5), 225-234. https://doi.org/10.1159/000029094 
Suhail, K., \& Cochrane, R. (2002). Effect of Culture and Environment on the Phenomenology of Delusions and Hallucinations. International Journal of Social Psychiatry, 48(2), 126-138. https://doi.org/10.1177/002076402128783181

Tanenberg-Karant, M., Fennig, S., Ram, R., Krishna, J., Jandorf, L., \& Bromet, E. J. (1995). Bizarre delusions and first-rank symptoms in a first-admission sample: A preliminary analysis of prevalence and correlates. Comprehensive Psychiatry, 36(6), 428-434. https://doi.org/10.1016/S0010-440X(95)90250-3

Tomasello, M. (2009). Why we cooperate. MIT press.

Tooby, J., \& Cosmides, L. (2010). Groups in mind: The coalitional roots of war and morality. Human Morality and Sociality: Evolutionary and Comparative Perspectives, 91-234.

Trainor, B. C. (2011). Stress responses and the mesolimbic dopamine system: Social contexts and sex differences. Hormones and Behavior, 60(5), 457-469. https://doi.org/10.1016/j.yhbeh.2011.08.013

Tripoli, G., Quattrone, D., Ferraro, L., Gayer-Anderson, C., Rodriguez, V., Cascia, C. L., ... Forti, M. D. (2019). Jumping To Conclusions, General Intelligence, And Psychosis Liability: Findings From The Multicentric EU-GEI Case-Control Study. BioRxiv, 634352. https://doi.org/10.1101/634352

van Ommen, M. M., van Laar, T., Cornelissen, F. W., \& Bruggeman, R. (2019). Visual hallucinations in psychosis. Psychiatry Research, 280, 112517. https://doi.org/10.1016/j.psychres.2019.112517

Ventura, J., Wood, R. C., \& Hellemann, G. S. (2013). Symptom Domains and Neurocognitive Functioning Can Help Differentiate Social Cognitive Processes in Schizophrenia: A Meta-Analysis. Schizophrenia Bulletin, 39(1), 102-111. https://doi.org/10.1093/schbul/sbr067 
Voce, A., Calabria, B., Burns, R., Castle, D., \& McKetin, R. (2019). A Systematic Review of the Symptom Profile and Course of Methamphetamine-Associated Psychosis. Substance Use \& Misuse, 54(4), 549-559. https://doi.org/10.1080/10826084.2018.1521430

Von Domarus, E. (1944). The specific laws of logic in schizophrenia. In Language and thought in schizophrenia (pp. 104-113).

Wardle, M. C., \& de Wit, H. (2012). Effects of amphetamine on reactivity to emotional stimuli. Psychopharmacology, 220(1), 143-153. https://doi.org/10.1007/s00213-0112498-7

Wardle, M. C., Garner, M. J., Munafò, M. R., \& de Wit, H. (2012). Amphetamine as a social drug: Effects of d-amphetamine on social processing and behavior. Psychopharmacology, 223(2), 199-210. https://doi.org/10.1007/s00213-012-2708-y

Warren, N., O’Gorman, C., Hume, Z., Kisely, S., \& Siskind, D. (2018). Delusions in Parkinson's Disease: A Systematic Review of Published Cases. Neuropsychology Review, 28(3), 310-316. https://doi.org/10.1007/s11065-018-9379-3

Winton-Brown, T. T., Fusar-Poli, P., Ungless, M. A., \& Howes, O. D. (2014). Dopaminergic basis of salience dysregulation in psychosis. Trends in Neurosciences, 37(2), 85-94. https://doi.org/10.1016/j.tins.2013.11.003

Woods, A., Jones, N., Alderson-Day, B., Callard, F., \& Fernyhough, C. (2015). Experiences of hearing voices: Analysis of a novel phenomenological survey. The Lancet Psychiatry, 2(4), 323-331. https://doi.org/10.1016/S2215-0366(15)00006-1

Yager, J. A., \& Ehmann, T. S. (2006). Untangling Social Function and Social Cognition: A Review of Concepts and Measurement. Psychiatry: Interpersonal and Biological Processes, 69(1), 47-68. https://doi.org/10.1521/psyc.2006.69.1.47 